\title{
Variabilidade genética para caracteres morfométricos de matrizes de castanha-do-brasil da Amazônia Mato- grossense
}

\author{
Flora Ferreira CAMARGO ${ }^{1}$, Reginaldo Brito da COSTA², Marcos Deon Vilela de RESENDE ${ }^{3}$, Raul Alffonso \\ Rodrigues ROA ${ }^{4}$, Natasha Brianez RODRIGUES 5 , Leonardo Vivaldini dos SANTOS ${ }^{6}$, Ana Carla Almeida de \\ FREITAS
}

\begin{abstract}
RESUMO
O presente estudo objetivou estudar a variabilidade genética de matrizes de Bertholletia excelsa através da estimação de parâmetros e ganhos genéticos para os caracteres peso/ouriço $(\mathrm{g})$, peso de sementes/ouriço $(\mathrm{g})$ e número de sementes/ouriço no prémelhoramento da espécie. Foram utilizadas 90 matrizes de polinização aberta, sendo 30 matrizes de cada tipo, denominadas localmente de rajada, mirim e rosa, no município de Cotriguaçu, noroeste de Mato Grosso, regiáo amazônica. O experimento foi estabelecido sob delineamento inteiramente ao acaso, com 90 tratamentos (matrizes) e seis ouriços por matriz, com suas respectivas sementes. As variáveis foram analisadas usando-se a metodologia de modelo linear misto do software SELEGENREML/BLUP. Os coeficientes de herdabilidades individuais no sentido amplo dos efeitos genotípicos totais $(0,21,0,14 \mathrm{e}$ $0,34)$ para os caracteres peso/ouriço $(\mathrm{g})$, peso de sementes/ouriço $(\mathrm{g})$ e número de sementes/ouriço, respectivamente, sáo considerados moderados para os dois primeiros caracteres e alto para o caráter número de sementes/ouriço, sugerindo expressivo controle genético. A seleção das 10 melhores matrizes revelou predominância da procedência do tipo rosa, proporcionando ganhos genéticos expressivos de pelo menos $24,16 \%$ para peso/ouriço $(\mathrm{g}), 27,44 \%$ para peso de sementes/ouriço e $16,92 \%$ para o caráter número de sementes por ouriço. Os valores expressivos das matrizes do tipo rosa estimulam a utilização desses germoplasmas em programas de melhoramento genético da espécie, na seqüência das avaliaçôes, bem como apontando para a possibilidade de obtenção de híbridos intraespecíficos para caracteres desejáveis.
\end{abstract}

PALAVRAS-CHAVE: Bertholletia excelsa, parâmetros genéticos, ganho com seleção, Amazônia.

\section{Genetic variability for morphometric characteristics in brazilian nut parent trees from northern Mato Grosso, Amazon rain forest}

\begin{abstract}
The goal of the study was to detect genetic variability in Brazilian nuts parent trees through parameters estimation and genetic gains for the following indexes: fruit weight $(\mathrm{g})$ seed weight per fruit $(\mathrm{g})$ and number of seeds per fruit at species preimprovement. Ninety (90) open pollination parent trees were used, 30 of which being of each type, locally called "rajada", "mirim" and "rosa" at the Cotriguacu municipality, Northeastern of Mato Grosso State. A completely randomized design was used, with 90 treatments (parent trees) and six fruits per parcel with their respective seeds. Variables were analyzed using the method of mixed univariate additive linear model from the software SELEGEN-REML/BLUP. Individual heritability coefficients in the broad sense for total genotypic effects $(0,21,0,14$ e 0,34$)$ for fruit weight $(\mathrm{g})$ seed weight per fruit $(\mathrm{g})$ and number of seeds per fruit (g) respectively, are considered moderate for the two first indexes and high for number of seeds per fruit, suggesting substantial genetic control. Selection of the 10 best provenances and parent trees showed predominance of the "rosa" type, allowing substantial genetic gains of at least $24,16 \%$ for fruit weight (g), 27,44\% for seed weight per fruit and $16,92 \%$ for the number of seeds per fruit. The remarkable values for the "rosa" type suggest utilization of such germoplasms in genetic improvement programs of this species in the following evaluations. The results also point out the possibility of obtaining intraspecific hybrids for desirable characteristics.
\end{abstract}

KEY WORDS: Bertholletia excelsa, genetic parameters, gains with selection, Amazon.

\footnotetext{
1 Universidade Federal de Mato Grosso, E-mail: florafloresta@gmail.com

2 Universidade Federal de Mato Grosso, E-mail: reg.brito.costa@gmail.com

${ }^{3}$ Universidade Federal de Viçosa, Email: marcos.deon@gmail.com

${ }^{4}$ Universidade Estadual Paulista Julio de Mesquita Filho (UNESP), E-mail: geneticraul@ig.com.br

${ }^{5}$ Universidade Federal de Mato Grosso, E-mail: natashabrianez@gmail.com

${ }^{6}$ Universidade Federal de Mato Grosso, E-mail: rasbimbol@hotmail.com

${ }^{7}$ Universidade Federal de Mato Grosso, E-mail: anaubiracema@hotmail.com
} 


\section{INTRODUÇÃO}

A castanha-do-brasil (Bertholletia excelsa H.B.K) é uma espécie arbórea pertencente à família Lecythidaceae. A sua distribuição geográfica é ampla e abrange a Venezuela, Colômbia, Peru, Bolívia e Guiana. Porém, as formações de florestas mais densas ocorrem no Brasil (Lorenzi 2000).

Após a decadência da borracha, a extração da castanha-dobrasil passou a constituir o principal produto extrativo para exportação da regiấo. Com a contínua fragmentação das áreas nativas, os castanhais foram, em grande parte, substituídos por rodovias e ferrovias, deslocamento de migrantes, obras de infraestrutura, criação de novos municípios, expansão da pecuária e extração de madeiras. Como se trata de uma espécie alógama, necessitando, portanto de polinização cruzada para que ocorra a frutificação, os desmatamentos e as queimadas, ao destruírem o habitat natural do agente polinizador (Hymenoptero do gênero Bombus spp.), têm contribuído para a redução de sua produção (Homma e Menezes 2008).

A sua utilização vai além do uso como alimento, que é ingerido in natura ou industrializado. Segundo Souza (2006), a espécie também é fonte de matéria-prima na produção de artesanatos (luminárias, cinzeiros, colares, chocalhos, etc.), cosméticos (sabonetes, xampus, creme hidratantes, óleo e batom) e fármacos (repelente, cicatrizantes, etc.).

Devido à castanha-do-brasil constituir-se em um produto da atividade especialmente extrativista e a diminuição das áreas nativas disponíveis, torna-se necessária a introdução de castanhais cultivados. Dessa forma, segundo Tonini et al. (2008), é imprescindível o desenvolvimento de pesquisas que procurem quantificar a produção de frutos por árvore, e determinar quais são os fatores que influenciam a variabilidade da produção individual.

Estudos relacionados à produção volumétrica e variáveis morfométricas têm sido realizados para a espécie, conforme dados obtidos por Ferreira e Tonini (2009) e Costa et al. (2009). Porém, estudos mais detalhados e considerando avaliaçóes genéticas devem ser conduzidos, visando avaliar o controle genético sobre a produtividade (Fernandes 2007). Os dados relativos à morfometria de frutos podem revelar variabilidade fenotípica dentro e entre as populaçôes (Carvalho 2008) ou tipologias da espécie.

A despeito da importância econômica e da ampla utilização da espécie, são incipientes as informaçôes sobre diversos aspectos, sendo um deles a variabilidade genética de suas matrizes no que se refere à morfologia de frutos e sementes, por meio de testes usando-se os germoplasmas que poderão subsidiar o estabelecimento de programas de melhoramento e conservação genética.

Nesse sentido, os testes de progênies, instrumentos importantes para o trabalho do melhorista, têm sido usados na estimativa de parâmetros genéticos e seleção de indivíduos, quando se procura avaliar a magnitude e a natureza da variância genética disponível, com vistas a quantificar e maximizar os ganhos genéticos, utilizando-se procedimento de seleção adequado.

Neste contexto, o presente estudo objetivou estudar a variabilidade genética de castanha-do-brasil, através da estimação dos parâmetros (herdabilidades individual no sentido amplo e da média de matrizes, variância fenotípica individual, coeficiente de determinação dos efeitos de tipos, média geral dos caracteres, coeficiente de variação genotípica, coeficiente de variação residual) e ganhos genéticos para os caracteres peso do ouriço (fruto) (g), peso de sementes/ouriço (g) e número de sementes/ouriço em matrizes de diferentes tipos de frutos da espécie, denominadas localmente de rosa (frutos maiores), rajada (frutos médios) e mirim (frutos menores).

\section{MATERIAL E MÉTODOS}

As coletas dos ouriços foram realizadas de 90 matrizes de polinização aberta, sendo 30 matrizes de cada tipo de fruto, denominadas localmente de rajada (1), mirim (2) e rosa (3), na Fazenda Sáo Nicolau, pertencente à Office National des Forêts (ONF Brasil), filial brasileira da ONF Internacional, localizada no município de Cotriguaçu, noroeste de Mato Grosso, em ambiente de domínio morfoclimático amazônico. As características da área de coleta estão contidas na Tabela 1 .

Tabela 1 - Detalhamento da área de coleta do material de estudo das matrizes, na Fazenda São Nicolau, município de Cotriguaçu, Estado de Mato Grosso.

\begin{tabular}{lc}
\hline Variáveis Descritivas & Município de Cotriguaçu \\
\hline Atitude $(\mathrm{m})$ & 254 \\
Clima (Köppen) & $\mathrm{Am}$ \\
Umidade relativa (\%) & $80 \mathrm{a} 85$ \\
Latitude & $9^{\circ} 47^{\prime} 51,32^{\prime \prime} \mathrm{S}$ \\
Longitude & $58^{\circ} 19^{\prime} 37,69^{\prime \prime} \mathrm{W}$ \\
Temperatura média anual $\left({ }^{\circ} \mathrm{C}\right)$ & 25 \\
Precipitação média anual $(\mathrm{mm})$ & 2000 \\
Classe de solo & Alissolos \\
Relevo & Ap3* \\
\hline
\end{tabular}

* suave dissecação (Governo do Estado de Mato Grosso 2000).

O experimento foi estabelecido sob delineamento inteiramente ao acaso, com 90 tratamentos (matrizes) e seis ouriços por matriz, com suas respectivas sementes.

Após a coleta, os ouriços foram transportados até o laboratório de sementes da Universidade Federal de Mato Grosso (UFMT), para as análises morfométricas. Os caracteres avaliados foram: a) peso do ouriço (g); b) peso de sementes/ ouriço $(\mathrm{g})$; c) número de sementes/ouriço. 
As variáveis foram analisadas usando-se a metodologia de modelo linear misto univariado genotípico do software SELEGEN-REML/BLUP (restricted maximum likelihood) apresentado por RESENDE (2007), consistindo do seguinte:

$\mathrm{y}=\mathrm{Xb}+\mathrm{Zg}+\mathrm{Wp}+\mathrm{e}, \mathrm{em}$ que:

"y" é o vetor de dados, "b" é o vetor dos efeitos fixos (no caso ajustando apenas a média geral), "g" é o vetor dos efeitos genotípicos de matrizes (assumidos como aleatórios), "p" é o vetor dos efeitos dos diferentes tipos e "e" o vetor de erros ou resíduos (aleatórios). As letras maiúsculas (X, Z e W) representam as matrizes de incidência para os referidos efeitos.

Equaçóes de modelo misto

$$
\left[\begin{array}{ccc}
X^{\prime} X & X^{\prime} Z & X^{\prime} W \\
Z^{\prime} X & Z^{\prime} Z+A^{-1} \lambda_{1} & Z^{\prime} W \\
W^{\prime} X & W^{\prime} Z & W^{\prime} W+I \lambda_{2}
\end{array}\right]\left[\begin{array}{c}
\hat{b} \\
\hat{g} \\
\hat{p}
\end{array}\right]=\left[\begin{array}{c}
X^{\prime} y \\
Z^{\prime} y \\
W^{\prime} y
\end{array}\right] \text {, em que: }
$$

$\lambda_{1}=\frac{\hat{\sigma}_{e}^{2}}{\hat{\sigma}_{g}^{2}}=\frac{1-\hat{h}^{2}-\hat{p}^{2}}{\hat{h}^{2}} ; \quad \lambda_{2}=\frac{\hat{\sigma}_{e}^{2}}{\hat{\sigma}_{p}^{2}}=\frac{1-\hat{h}^{2}-\hat{p}^{2}}{\hat{p}^{2}}$

Os parâmetros genéticos e variâncias foram obtidas a partir do que segue:

$$
\hat{h}_{g}^{2}=\frac{\hat{\sigma}_{g}^{2}}{\hat{\sigma}_{g}^{2}+\hat{\sigma}_{p}^{2}+\hat{\sigma}_{e}^{2}}=\text { herdabilidade individual no }
$$

sentido amplo dos efeitos genotípicos de matrizes. Essa herdabilidade no sentido amplo a rigor equivale ao coeficiente de repetibilidade, o qual representa o limite superior que a herdabilidade no sentido amplo pode alcançar (Falconer e Mackay 1996).

$$
\hat{h}_{m m}^{2}=\frac{\hat{\sigma}_{g}^{2}}{\hat{\sigma}_{g}^{2}+\hat{\sigma}_{p}^{2} / p+\hat{\sigma}_{e}^{2} / n}=\text { repetibilidade média }
$$

de matrizes.

$\hat{\sigma}_{p}^{2}=$ variância entre os diferentes tipos;

$\hat{\sigma}_{e}^{2}=$ variância residual;

$\hat{\sigma}_{g}^{2}=$ variância genotípica;

$c_{\text {proc }}^{2}=$ coeficiente de determinação do efeito dos diferentes tipos;

$\hat{r}_{g g}=\sqrt{\hat{h}_{m m}^{2}}=$ Acurácia seletiva;

$\mathrm{CV}_{\mathrm{gi}}(\%)=\frac{\sqrt{\hat{\sigma}_{g}^{2}}}{\bar{X}} \cdot 100$

$\mathrm{CV}_{\mathrm{gi}}=$ coeficiente de variação genotípica entre matrizes;

$\mathrm{CV}_{\mathrm{e}}(\%)=\frac{\sqrt{p^{2}+\hat{\sigma}_{c} / n}}{\bar{X}} .100$
$\mathrm{CV}_{\mathrm{e}}=$ coeficiente de variação experimental;

O material botânico herborizado da espécie foi identificado, catalogado e depositado no herbário da Universidade Federal de Mato Grosso (UFMT) em Cuiabá e considerou-se a taxa de cruzamento para a espécie de $85 \%$, portanto com $15 \%$ de autofecundação, conforme O'Malley et al. (1988).

A utilização da metodologia SELEGEN-REML/BLUP, desenvolvida para o melhoramento de plantas perenes tem maximizado os ganhos genéticos com seleção (Resende 2002; Costa et al. 2005; Missio et al. 2005), por tratar-se de um procedimento estimativo, especialmente para dados desbalanceados, predizendo valores genéticos dos indivíduos em testes de progênies (Resende 2002).

\section{RESULTADOS E DISCUSSÃO}

Os resultados referentes às estimativas por tipo e suas respectivas médias genotípica para os caracteres peso do ouriço (g), peso de sementes por ouriço $(\mathrm{g})$ e número de sementes por ouriço das matrizes são apresentados na Tabela 2.

Constata-se que as matrizes do tipo 3 apresentaram-se expressivas para os valores de médias genotípicas para todos os caracteres morfométricos. Os resultados demonstram que as matrizes deste tipo, com seus ouriços (frutos) maiores deve

Tabela 2 - Média genotípica dos tipos para os caracteres peso do ouriço $(\mathrm{g})$, peso de sementes por ouriço $(\mathrm{g})$ e número de sementes por ouriço de castanha-do-brasil, na Fazenda São Nicolau, município de Cotriguaçu, MT.

\begin{tabular}{lcccc}
\hline & & \multicolumn{3}{c}{ Variáveis } \\
\cline { 3 - 5 } Ordem & Tipos* $^{*}$ & $\begin{array}{c}\text { Peso de } \\
\text { Ouriço }(\mathrm{g})\end{array}$ & $\begin{array}{c}\text { Peso de } \\
\text { Sementes } \\
\text { por Ouriço } \\
(\mathrm{g})\end{array}$ & $\begin{array}{c}\text { Número de } \\
\text { Sementes } \\
\text { por Ouriço }\end{array}$ \\
\hline 1 & 3 & 697,13 & 124,5 & 19,86 \\
2 & 1 & 481,37 & 119,93 & 16,51 \\
3 & 2 & 434,35 & 118,66 & 15,17 \\
\hline
\end{tabular}

${ }^{1}$ Tipos: 1 - rajada; 2 - mirim; 3 - rosa.

ser considerada no processo de coleta e beneficiamento da castanha na área de abrangência do estudo.

Os resultados referentes às estimativas de parâmetros genotípico para os caracteres peso de Ouriço (g), peso de sementes $(\mathrm{g})$ por ouriço e número de sementes por ouriço em matrizes de castanha-do-brasil das matrizes são apresentados na Tabela 3.

Os coeficientes de herdabilidades individuais no sentido amplo, dos efeitos genotípicos totais $(0,21,0,14$ e 0,34$)$ para os caracteres peso/ouriço (g), peso de sementes/ouriço (g) e número de sementes/ouriço, respectivamente, são considerados moderados para os dois primeiros caracteres e alto para o caráter 
Tabela 3 - Estimativas de parâmetros genotípicos para os caracteres peso de Ouriço $(\mathrm{g})$, peso de sementes $(\mathrm{g})$ por ouriço e número de sementes por ouriço em matrizes de castanha-do-brasil, na Fazenda São Nicolau, município de Cotriguaçu, MT.

\begin{tabular}{lccc}
\hline Estimativas $^{1}$ & Peso de Ouriço (g) & $\begin{array}{c}\text { Peso de Sementes } \\
(\mathrm{g}) \text { por ouriço }\end{array}$ & $\begin{array}{c}\text { Número de } \\
\text { sementes por } \\
\text { ouriço }\end{array}$ \\
\hline$\hat{h}_{g}^{2}$ & 0,21 & 0,14 & 0,34 \\
$\hat{h}_{m m}^{2}$ & 0,62 & 0,58 & 0,54 \\
$\hat{\sigma}_{f}^{2}$ & 29763,72 & 2798,06 & 14,57 \\
$c_{\text {proc }}^{2}$ & 0,67 & 0,75 & 0,38 \\
Média geral $^{2}$ & 537,62 & 121,03 & 17,18 \\
$\left(\mathrm{CV}_{\mathrm{gi}} \%\right)$ & 14,57 & 16,57 & 12,89 \\
$\left(\mathrm{CV}_{\mathrm{e}} \%\right)$ & 28,59 & 40,44 & 18,10 \\
\hline
\end{tabular}

${ }^{1}$ Herdabilidade individual no sentido amplo dos efeitos genotípicos de matrizes $\left(\hat{h}_{g}^{2}\right.$ ), herdabilidade da média de matrizes $\left(\hat{h}_{m m}^{2}\right)$, variância fenotípica individual $\left(\hat{\sigma}^{2}\right)$, coeficiente de determinação dos efeitos de diferentes tipos $\left(c_{\text {proc }}^{2}\right)$, média geral dos caracteres, coeficiente de variação genotípica $\left(\mathrm{CV}_{\mathrm{gi}} \%\right)$ e 0 coeficiente de variação residual ( $\left.\mathrm{CV}_{\mathrm{e}} \%\right)$.

número de sementes/ouriço, sugerindo expressivo controle genético. Portanto, as estimativas obtidas poderão maximizar os ganhos genéticos com seleção na seqüência das avaliaçóes.

A mais importante função da herdabilidade no estudo genético do caráter métrico é o seu papel preditivo expressando a confiança do valor fenotípico como um guia para o valor genético, ou o grau de correspondência entre o valor fenotípico e o valor genético (Falconer 1987; Vencovsky e Barriga 1992). Segundo Falconer (1987) a herdabilidade é uma propriedade não somente de um caráter, mas também da população e das circunstâncias de ambientes às quais os indivíduos estão sujeitos. $\mathrm{O}$ valor da herdabilidade poderá ser afetado se houver alteração em qualquer um dos componentes da variância.

Os valores encontrados para as estimativas dos coeficientes de determinação dos efeitos dos diferentes tipos $\left(c_{\text {proc }}^{2}\right)$, demonstrando que a principal variação foi entre os tipos e não dentro dos diferentes tipos. Essas assertivas são condizentes com o que está contido na Tabela 2, que demonstra a variação entre os tipos. Segundo Martinez (2006), as estimativas dos coeficientes de determinação dos efeitos de procedências (neste caso, tipos) quando apresentam baixas magnitudes, tendem a maior variabilidade dentro dos tipos do que entre os diferentes tipos.

Os coeficientes de variação genética individual $\left(\mathrm{CV}_{\mathrm{gi}} \%\right)$, que expressam em percentagem da média geral a quantidade de variação genética existente, apresentaram 14,57 16,57 12,89 para os caracteres peso/ouriço (g), peso de sementes/ouriço (g) e número de sementes/ouriço, respectivamente. Esses valores sugerem que, em futuras avaliaçóes de campo, poderá haver maior expressão da variação genética para os caracteres estudados.

Os resultados referentes às matrizes dos diferentes tipos, efeitos genotípicos preditos, valores genotípicos, ganhos genéticos em gramas, nova média em gramas e acurácia seletiva para o caráter peso de ouriço $(\mathrm{g})$ de castanha-do-brasil são apresentados na Tabela 4.

Observa-se que as matrizes do tipo 3 predominaram amplamente sobre as matrizes dos dois outros tipos, apresentando as matrizes 25 e 17 com os melhores desempenhos, mostrando-se promissoras, em relação aos ganhos genéticos, que variaram de 129,90 g a 277,78 g

Tabela 4 - Tipos, matrizes, efeitos genotípicos preditos, valores genotípicos, ganhos genéticos em gramas, nova média em gramas e acurácia seletiva para o caráter peso de ouriço (g) de castanha-do-brasil, na Fazenda São Nicolau, município de Cotriguaçu, MT.

\begin{tabular}{|c|c|c|c|c|c|c|}
\hline Ordem & Tipos & Matriz & $\begin{array}{c}\text { Efeito } \\
\text { genotípico } \\
\text { predito }\end{array}$ & $\begin{array}{c}\text { Valores } \\
\text { genotípicos }\end{array}$ & $\begin{array}{c}\text { Ganho } \\
\text { genético } \\
\text { (gramas) }\end{array}$ & $\begin{array}{c}\text { Nova } \\
\text { Média } \\
\text { (gramas) }\end{array}$ \\
\hline 1 & 3 & 25 & 277,78 & 815,40 & 277,78 & 815,40 \\
\hline 2 & 3 & 17 & 187,06 & 724,68 & 232,42 & 770,04 \\
\hline 3 & 3 & 28 & 141,70 & 679,32 & 202,18 & 739,80 \\
\hline 4 & 3 & 27 & 117,51 & 655,13 & 181,01 & 718,63 \\
\hline 5 & 2 & 9 & 110,96 & 648,58 & 167,00 & 704,62 \\
\hline 6 & 2 & 15 & 107,94 & 645,56 & 157,16 & 694,78 \\
\hline 7 & 3 & 29 & 96,34 & 633,96 & 148,47 & 686,09 \\
\hline 8 & 1 & 2 & 92,50 & 630,12 & 141,47 & 679,09 \\
\hline 9 & 2 & 10 & 83,75 & 621,36 & 135,06 & 672,68 \\
\hline 10 & 1 & 6 & 83,43 & 621,05 & 129,90 & 667,51 \\
\hline \multicolumn{6}{|c|}{ Acurácia Seletiva $\left(\hat{r}_{g g}\right)$} & 0,45 \\
\hline
\end{tabular}

${ }^{2}$ Valores genotípicos $=\mu+\mathrm{g}$

elevando a nova média do tipo em pelo menos $24,16 \%$ para o caráter peso de ouriço $(\mathrm{g})$. A acurácia seletiva encontrada para o caráter $(0,45)$ é considerada moderada para as condiçóes experimentais. Conforme Cargnelutti-Filho e Storck (2009), em geral ensaios com maior acurácia seletiva, são aqueles com menor variância residual e maior variância genética.

Os resultados referentes aos tipos, matrizes, efeitos genotípicos preditos, valores genotípicos, ganhos genéticos em gramas, nova média em gramas e acurácia seletiva para o caráter peso de sementes por ouriço (g) de castanha-do-brasil são apresentados na Tabela 5 .

De maneira similar, constata-se que os indivíduos do tipo 3 predominaram amplamente sobre os indivíduos dos dois outros tipos, apresentando as matrizes 29, 26 e 25 com os melhores desempenhos, mostrando-se promissoras, em relação aos ganhos genéticos, que variaram de 33,22 g a 58,99 g elevando a nova média em pelo menos $27,44 \%$ para o caráter 
Tabela 5 - Tipos, matrizes, efeitos genotípicos preditos, valores genotípicos, ganhos genéticos em gramas, nova média em gramas e acurácia seletiva para o caráter peso de sementes por ouriço (g) de castanha-do-brasil, na Fazenda São Nicolau, município de Cotriguaçu, MT.

\begin{tabular}{lcccccc}
\hline Ordem & Tipos & Matriz & $\begin{array}{c}\text { Efeito } \\
\text { genotípico } \\
\text { predito }(\mathrm{g})\end{array}$ & $\begin{array}{c}\text { Valores } \\
\text { genotípicos } \\
(\mathrm{u}+\mathrm{g})\end{array}$ & $\begin{array}{c}\text { Ganho } \\
\text { genético }\end{array}$ & $\begin{array}{c}\text { Nova } \\
\text { Média }\end{array}$ \\
\hline 1 & 3 & 29 & 58,99 & 180,01 & 58,99 & 180,01 \\
2 & 3 & 26 & 41,00 & 162,03 & 49,99 & 171,02 \\
3 & 3 & 25 & 34,01 & 155,04 & 44,66 & 165,69 \\
4 & 2 & 21 & 31,76 & 152,79 & 41,44 & 162,47 \\
5 & 1 & 14 & 30,77 & 151,80 & 39,31 & 160,33 \\
6 & 2 & 2 & 28,86 & 149,89 & 37,57 & 158,59 \\
7 & 3 & 24 & 28,36 & 149,39 & 36,25 & 157,28 \\
8 & 1 & 27 & 26,90 & 147,93 & 35,08 & 156,11 \\
9 & 1 & 10 & 26,01 & 147,04 & 34,07 & 155,10 \\
10 & 2 & 10 & 25,52 & 146,55 & 33,22 & 154,25 \\
\hline \multicolumn{7}{l}{ Acurácia Seletiva $\left(\hat{r}_{\mathrm{gg}}\right)$} \\
\hline \multicolumn{7}{l}{} \\
\hline
\end{tabular}

peso de sementes por ouriço (g). A acurácia seletiva encontrada para o caráter $(0,38)$ também é considerada moderada para as condiçóes experimentais.

Os resultados referentes aos tipos, matrizes, efeitos genotípicos preditos, valores genotípicos, ganhos genéticos em gramas, nova média em gramas e acurácia seletiva para o caráter número de sementes por ouriço (g) de castanha-dobrasil são apresentados na Tabela 6 .

A acurácia seletiva encontrada para o caráter $(0,58)$ é considerada elevada para as condiçôes experimentais. Segundo Van Vleck et al. (1987), os valores preditos permitem indicar com mais segurança que o germoplasma em estudo maximizará as possibilidades de progresso genético com

Tabela 6 - Tipos, matrizes, efeitos genotípicos preditos, valores genotípicos, ganhos genéticos em gramas, nova média em gramas e acurácia seletiva para o caráter número de sementes por ouriço de castanha-do-brasil, na Fazenda São Nicolau, município de Cotriguaçu, MT.

\begin{tabular}{lcccccc}
\hline Ordem & Tipos & Matriz & $\begin{array}{c}\text { Efeito } \\
\text { genotípico } \\
\text { predito }(\mathrm{g})\end{array}$ & $\begin{array}{c}\text { Valores } \\
\text { genotípicos } \\
(\mathrm{u}+\mathrm{g})\end{array}$ & $\begin{array}{c}\text { Ganho } \\
\text { genético }\end{array}$ & $\begin{array}{c}\text { Nova } \\
\text { Média }\end{array}$ \\
\hline 1 & 2 & 26 & 5,52 & 22,70 & 5,52 & 22,70 \\
\hline 2 & 2 & 2 & 5,09 & 22,26 & 5,30 & 22,48 \\
3 & 1 & 29 & 3,46 & 20,64 & 4,69 & 21,87 \\
\hline 4 & 3 & 27 & 3,23 & 20,41 & 4,33 & 21,51 \\
5 & 1 & 27 & 3,17 & 20,35 & 4,10 & 21,27 \\
6 & 3 & 29 & 3,09 & 20,26 & 3,93 & 21,11 \\
7 & 2 & 16 & 3,04 & 20,22 & 3,80 & 20,98 \\
\hline 8 & 1 & 14 & 2,88 & 20,06 & 3,69 & 20,86 \\
9 & 3 & 26 & 2,79 & 19,97 & 3,59 & 20,77 \\
10 & 2 & 21 & 2,75 & 19,93 & 3,50 & 20,68 \\
\hline \multicolumn{7}{l}{ Acurácia Seletiva $\left(\hat{r}_{\mathrm{gg} g}\right)$} \\
\hline
\end{tabular}

seleção. De maneira geral, os valores genéticos preditos não são iguais aos valores genéticos verdadeiros dos indivíduos. A proximidade entre estes dois valores pode ser avaliada com base na estatística denominada acurácia. A literatura prática pertinente ao assunto apresentada por Resende et al. (1995) e Costa et al. (2000) tem demonstrado a importância da acurácia para apontar o grau de confiabilidade dos resultados obtidos na avaliação genética.

Os resultados obtidos para o caráter número de sementes apresentaram um equilíbrio maior, com os tipos 1 e 2 aparecendo com destaque entre as 10 melhores, o que demonstra que, embora o peso de suas sementes não seja semelhante ao do tipo 3, os ouriços contém um número maior de sementes. Neste sentido, estudos mais detalhados devem ser realizados sobre a qualidade das sementes dos tipos 1 e 2 .

Dessa forma, as matrizes 26 e 2 (tipo 2), e 29 (tipo 1), constituem as 3 de melhor desempenho entre as 10 melhores matrizes para o caráter número de sementes por ouriço, mostrando-se promissoras em relação aos ganhos genéticos, que variaram de 3,50 e 5,52 elevando a nova média em pelo menos $16,92 \%$ para o caráter.

De maneira geral, os valores expressivos do tipo rosa (3) estimulam a utilização desses germoplasmas em programas de melhoramento genético da espécie, na seqüência das avaliaçóes em viveiro e no campo. Apontam para a possibilidade de obtenção de híbridos intraespecíficos para caracteres desejáveis entre os diferentes tipos de melhor desempenho, bem como para a necessidade de trabalhos taxonômicos para os diferentes germoplasmas que possibilitem a caracterização de possíveis variedades da espécie da castanha-do-brasil na regiáo de estudo.

Além disso, os resultados obtidos no pré-melhoramento poderáo subsidiar o estabelecimento de um programa de melhoramento genético da espécie, associado à implementação de planos de manejo florestal para a castanha-do-brasil na região, gerando efeitos positivos para conservação do ecossistema florestal regional e para sustentabilidade do sistema extrativista.

\section{AGRADECIMENTOS}

Os autores agradecem à ONF Brasil por todo o apoio logístico durante a coleta do material genético e a CAPES e $\mathrm{CNPq}$ pelas bolsas concedidas.

\section{BIBLIOGRAFIA CITADA}

Cargnelutti-Filho, A.; Storck, L. 2009. Measures of experimental precision degree in corn cultivar competition trials. Pesquisa Agropecuária Brasileira, 44(2): 111-117 (in Portuguese, with abstract in English).

Carvalho, P.C.L.; Ritzinger, R.; Soares-Filho, W.S.; Ledo, C.A.S. 2008. Morphological physical and chemical characteristics of 
fruits of umbu-cajazeira populations in the State of Bahia. Revista Brasileira de Fruticultura, 30 (1): 140-147 (in Portuguese, with abstract in English).

Costa, J.R.; Castro, A.B.C.; Wandelli, E.V.; Coral, S.C.T.; Souza, S.A.G. 2009. Silvicultural aspects of Brazil nut (Bertholletia excelsa) in agroforestry systems in Central Amazonia. Acta Amazonica, 39(4): 843-850 (in Portuguese, with abstract in English).

Costa, R. B.; Resende, M.D.V.; Contini, A.Z.; Rego, F.L.H.; Roa, R.A.R.; Martins, W.J. 2005. Genetic evaluation of individuals of erva-mate (Ilex paraguariensis St. Hil.) progenies, in the Caarapó/ MS region, using the REMLBLUP procedure. Ciência Florestal, 15: 371-376 (in Portuguese, with abstract in English).

Costa, R.B.; Resende, M.D.V. de; Gonçalves, P. de S.; Higa, A.R. 2000. Selection and genetic gain in populations of Hevea brasiliensis with a mixed mating system. Genetics and Molecular Biology, 23: 671-679.

Falconer, D.S. 1987. Introduction to quantitative genetics. Viçosa, UFV. 279 pp (in Portuguese).

Falconer, D. S.; Mckay, T. F. C. 1996. Introduction to quantitative genetics. Malasya, Longman. 464 pp.

Fernandes, E.T.M. Morphological Diversity and Production of Bertholletia excelsa H.B.K. (Lecythidaceae) in the Southeast of Acre - Brazil. Anais do VIII Congresso de Ecologia do Brasil, 1: 27-28 (in Portuguese).

Ferreira, L.M.M.; Tonini, H. 2009. Behavior of castanha-dobrasil (Bertholletia excelsa) and cupiúba (Goupia glabra) in an agrosilvicultural system in Confiança region, Cantá - Roraima State. Acta Amazonica, 39(4): 835-842 (in Portuguese, with abstract in English).

Governo do Estado de Mato Grosso. 2000. Socio-economic-ecological Zoning: socio -economic-ecological diagnostic of the Mato Grosso State e technical assistance on the formulation of the $2^{\circ}$ approximation. Cuiabá, MT. 29 pp (in Portuguese).

Homma, A.K.O.; Menezes, A.J.E.A. 2008. Evaluation of a Brazilnut Beneficiation Industry, in the Microregion of Cametá, PA. Comunicado Técnico 213, Belém. 10 pp (in Portuguese).

Lorenzi, H. 2000. Brazilian Trees: A Guide to the Identification and Cultivation of Brazilian Native Trees, Nova Odessa, Plantarum, 368 pp (in Portuguese).
Martinez, D.T. 2006. Genetic Selection of Acacia mearnsii de wild. (acácia-negra), aiming to increase wood and tannin quality and production, in Rio Grande do Sul. Dissertação de Mestrado, Universidade Federal do Paraná, Curitiba. 2006. 90 pp (in Portuguese, with abstract in English).

Missio, R.F.; Silva, A.M.; Dias, L.A.S.; Moraes, M.L.T.; Resende, M.D.V. 2005. Estimates of genetic parameters and prediction of additive genetic values in Pinus kesya progenies. Crop breeding and applied biotechnology, 5: 394-401.

O’Malley, D.M.; Buckley, D.P.; Prance, G.T.; Bawa, K.S. 1988. Genetics of Brazil nut (Bertholletia excelsa Humb. \& Bonpl.: Lecythidaceae). Theoretical and Applied Genetics, 76: 929-932.

Resende, M.D.V. 2002. Biometrical Genetics and Statistics in Perennial Plant Breeding. Brasília, Embrapa Informação Tecnológica. 975 pp (in Portuguese).

Resende, M.D.V. 2007. Selegen-Reml/Blup: statistical system and computerized genetic selection through linear mixed models. Colombo, Embrapa Florestas. 360 pp (in Portuguese).

Resende, M.D.V.; Araujo, A.J.; Sampaio, P.T.B.; Wiecheteck, M.S.S. 1995. Accuracy, confidence ranges and variance of response associated to 22 selection methods in Pinus caribaea var. hondurensis. Revista Floresta, 24: 35-45 (in Portuguese, with abstract in English).

Souza, I.F. 2006. Production chain of Brazil-nut (Bertholletia excelsa) in the State of Mato Grosso. Dissertaçáo de Mestrado, Universidade Federal de Mato Grosso do Sul, Campo Grande. 152 pp (in Portuguese, with abstract in English).

Tonini, H.; Kaminski, P.E.; Costa, P. 2008. Relationship of Brazil nut seed yield to crown morphometric characteristics and competition indexes. Pesquisa Agropecuária Brasileira, Brasília, 43(11): 1509-1516 (in Portuguese, with abstract in English).

Van Vleck, L.D.; Pollak, E.J.; Oltenacu, E.A.B. 1987. Genetics for the animal sciences. New York, W.H. Freeman, 391pp.

Vencovsky, R.; Barriga, P. 1992. Biometrical Genetics in the phyto improvement. Sociedade Brasileira de Genética, Ribeirão Preto, 496 pp (in Portuguese).

Recebido em 21/03/2010

Aceito em 31/05/2010 